\title{
Lentes de contato terapêuticas e as vantagens dos materiais de alto Dk
}

\author{
Therapeutic contact lenses and the advantages of high Dk materials
}

\author{
Cleusa Coral-Ghanem ${ }^{1}$ \\ Vinícius Coral Ghanem² \\ Ramon Coral Ghanem ${ }^{3}$
}

\section{RESUMO}

As lentes de contato terapêuticas são úteis para o tratamento de uma série de doenças da superfície ocular. Suas principais finalidades são: reduzir a dor; proteger a córnea; facilitar e manter a cicatrização epitelial; restaurar a câmara anterior e liberar medicamentos na superfície ocular. Há uma variedade de tipos e materiais, sendo que a escolha depende da doença a ser tratada, tempo de uso e necessidades fisiológicas do olho doente. Doenças como a ceratopatia bolhosa, erosão recorrente do epitélio corneano, olho seco e defeitos epiteliais pós-operatórios, além de várias outras doenças que afetam a superfície ocular podem ser tratadas com a utilização das lentes de contato terapêuticas. Elas não devem ser utilizadas em processos infecciosos ou em pacientes que estão impossibilitados de retornar para acompanhamento regular. A adaptação, controle e manutenção adequados das lentes de contato terapêuticas são fundamentais para prevenir complicações, como: neovascularização corneana, conjuntivite papilar gigante ou ceratite infecciosa. $\mathrm{O}$ controle e manutenção foram facilitados com o advento das lentes de contato terapêuticas de silicone-hidrogel. Essas lentes permitem trocas menos freqüentes devido a sua maior oxigenação e, em casos selecionados, aceleram a reepitelização corneana.

Descritores: Lentes de contato hidrofílicas; Hidrogéis; Silicones; Epitélio anterior/fisiologia; Cicatrização de feridas/fisiologia; Ceratite; Ceratectomia fotorrefrativa; Acuidade visual

Trabalho realizado no Hospital de Olhos Sadalla Amin Ghanem.

${ }^{1}$ Doutora em Ciências - Área de Concentração Oftalmologia - Faculdade de Medicina da Universidade de São Paulo - USP - São Paulo (SP) - Brasil. Responsável pelo Departamento de Lentes de Contato do Hospital de Olhos Sadalla Amin Ghanem - Joinville (SC) - Brasil.

${ }^{2}$ Doutor em Ciências pela Universidade de São Paulo USP- São Paulo (SP) - Brasil. Oftalmologista e Presidente da Comissão de Controle de Infecção Hospitlar do Hospital de Olhos Sadalla Amin Ghanem - Joinville (SC) - Brasil. Especialista com Residência em Oftalmologia na Universidade Estadual de Campinas - UNICAMP Campinas (SP) - Brasil. Fellow em Cirurgia Refrativa e Córnea na Universidade da Califórnia, Davis, EUA.

${ }^{3}$ Pós-graduando em Oftalmologia com Residência e Preceptoria no Hospital das Clínicas da Universidade de São Paulo - USP- São Paulo (SP) - Brasil. Oftalmologista do Hospital de Olhos Sadalla Amin GhanemJoinville (SC) - Brasil. Fellow em Cirurgia Refrativa e Córnea no Massachusetts Eye and Ear Infirmary - Harvard Medical School, Boston, MA, USA.

Endereço para correspondência: Cleusa Coral Ghanem. Rua Abdon Batista, 172 - Joinville (SC)

CEP 89201-010

E-mail: coral.joi@terra.com.br

\footnotetext{
INTRODUÇÃO

Nos anos 50, vislumbrou-se a possibilidade de fabricar lentes de contato (LC) a partir de hidroxi-etil-metacrilato polimerizado (HEMA), base para as LC hidrofílicas de hoje. O trabalho pioneiro com LC de HEMA foi realizado por Otto Withterle, Daroslav Lim e Maximillian Dreifus, em 1963.

As LC hidrofílicas foram disponibilizadas no mercado em 1971 e, em 1973, LC mais finas, de troca anual, passaram a ser indicadas com fins terapêuticos, necessitando de remoção freqüente. Depois vieram as LC de colágeno, para serem imersas em soluções oftálmicas, antes de colocar nos olhos; liberam o medicamento e dissolvem-se lentamente, num período de 12 a 72 horas. Em 1987, com a introdução das LC hidrofílicas descartáveis, estas passaram a ser as preferidas para finalidades terapêuticas. Em 1998 foi desenvolvido um material gelatinoso com alto Dk, o siliconehidrogel, que permite maior transmissibilidade ao $\mathrm{O}_{2}$ que o hidrofílico. Desde 1999, a LC de silicone-hidrogel tornou-se a primeira escolha como terapêutica, quando o objetivo é proteger e facilitar a recuperação do epitélio corneal ou para evitar ruptura de macrobolhas epiteliais na ceratopatia bolhosa.
} 
As lentes de contato terapêuticas (LCT) representam uma importante opção no tratamento e controle de numerosas doenças da superfície ocular. Têm como finalidades:

- Reduzir a dor causada por abrasões, erosões e úlceras corneais.

- Proteger a córnea que apresenta ceratite filamentar, neurotrófica, neuroparalítica e nos casos de ceratopatia bolhosa.

- Facilitar e manter a cicatrização epitelial.

- Proteger a córnea contra o ressecamento e contra danos mecânicos secundários a entrópio e triquíase.

- Restaurar a câmara anterior quando o colapso da mesma é determinado por uma pequena perfuração.

- Liberar medicamentos na superfície ocular.

- Melhorar o desconforto após sutura de córnea, transplante, cirurgia fotorrefrativa ou cirurgia de correção de problemas palpebrais.

\section{As indicações mais freqüentes são:}

Ceratopatia bolhosa - É uma das indicações mais comuns para LCT com finalidade de aliviar a dor, enquanto o paciente aguarda o transplante de córnea. Para evitar ou protelar a vascularização da córnea, recomenda-se utilizar LC siliconehidrogel de alto Dk. Nos olhos sem possibilidade de reabilitação visual não existe a preocupação com o crescimento de vasos; a prevenção de infecção é a razão maior do controle médico.

Erosão recorrente de córnea - A LCT proporciona grande alívio e conforto, reduzindo drasticamente os sinais e sintomas (dor, sensação de corpo estranho, lacrimejamento e fotofobia) que surgem quando o paciente acorda, principalmente após dormir durante várias horas. A LCT deve ser usada durante 2 a 3 meses para permitir a firme adesão do epitélio à membrana basal. Em casos de erosão de ápice, nos portadores de ceratocone, usa-se o sistema "piggyback", dando preferência à LC de silicone-hidrogel.

Olho seco - O uso de LCT deve ser reservado para os casos que não alcançam sucesso com a utilização de lágrimas artificiais, uso de ácidos graxos essenciais em apresentação oral (p.ex. óleo de linhaça) e a oclusão dos pontos lacrimais. É imprescindível levar em consideração que LCG adaptada num olho seco apresenta maior formação de depósitos e aumenta o risco de infecção. Pode-se usar LC hidrofílica fina e de baixa hidratação, que sofre menor evaporação, mas se utiliza a de alta hidratação quando se tem adequada suplementação de lágrimas artificiais, sem preservativo. Com o advento das LC silicone-hidrogel, estudos demonstraram melhora subjetiva dos sintomas em pacientes com olho seco ${ }^{(1)}$. Um deles acompanhou 278 usuários de LC hidrofílica com sintomas de olhos secos, readaptados com LC silicone-hidrogel (lotrafilcon A), por três anos. Os resultados mostraram redução da freqüência e da gravidade dos sintomas já com uma semana de uso, e os usuários mantiveram-se bem até o final do estudo ${ }^{(2)}$.

Perfuração corneal - Existe indicação para LCT quando a perfuração é pequena, de até $1 \mathrm{~mm}$ de diâmetro, e não mostra encarceramento de tecido uveal. A LC deve ser adaptada ligeiramente apertada para melhor ocluir o orifício e provocar edema de córnea, com a intenção de aproximar as bordas da lesão, facilitando a sua oclusão. Pode ser usada também sobre o adesivo de cianoacrilato para dar conforto.

Distrofia corneal - Algumas distrofias corneais estão associadas com irregularidades do epitélio, determinando a ruptura freqüente do mesmo (erosão recorrente), o que causa dor, sensação de corpo estranho, fotofobia e lacrimejamento. A LCT está indicada para aliviar os sintomas, mas deve ser reservada para os casos recorrentes que apresentam acentuado desconforto para o paciente.

Queimadura química - Após diminuir a intensa reação inflamatória inicial, uma LCT pode ser usada para promover a reepitelização, prevenir e tratar a ruptura freqüente do epitélio. Além disso, tem a finalidade de proteger a córnea do ressecamento e do trauma causado por anormalidades palpebrais. Indica-se a utilização de lágrima artificial e antibiótico tópico, sem preservativos, sobre a LCT.

Ceratite neurotrófica - A LCT pode ser útil na recuperação do epitélio, quando a oclusão e o uso de lágrimas artificiais não surtem o efeito necessário. A combinação de LCT e gotas de soro autólogo podem ser usadas com sucesso, embora depósitos de albumina possam ocorrer na superfície da $\mathrm{LC}^{(3)}$. Deve-se fazer um acompanhamento médico rigoroso desses olhos que, devido à ausência de sensibilidade corneal, podem apresentar complicações graves. O uso profilático de antibiótico tópico é recomendável.

Ceratite de Thygeson - A LCT pode promover conforto nos casos resistentes ao tratamento com lubrificantes e corticosteróides.

Cirurgia de córnea com excimer laser - A forte dor, que ocorre nas primeiras 24 a 48 horas de pós-operatório da ceratectomia fotorrefrativa (PRK - photorefractive keratectomy), tem sua intensidade consideravelmente reduzida com o uso de LCT. A LCT evita o toque da pálpebra superior com as terminações nervosas corneais, expostas pela ceratectomia fotorrefrativa, reduzindo a dor e a sensação de corpo estranho. De maneira similar, as LCT são utilizadas no pós-operatório de "crosslinking" do colágeno corneal, enquanto ocorre a reepitelização. No pós-laser in situ keratomileusis (LASIK) indica-se LC principalmente quando acontece erosão do epitélio. Nesse caso, recomenda-se a remoção logo que o epitélio esteja refeito para diminuir a possibilidade de infecção.

As contra-indicações mais importantes das LCT são: infecção ocular e impossibilidade do paciente retornar para seguimento adequado, o que o exporia a um alto risco de complicações. São contra-indicações relativas, relacionadas com a possibilidade de infecção: blefarite seborreica, anormalidades do sistema lacrimal, higiene pessoal pobre e a presença de bolha filtrante.

A forma de adaptar a LCT inicia com a obtenção das medidas ceratométricas ou topográficas para a escolha da curva base (CB). Caso não seja possível, utilizam-se as medidas do 
outro olho ou coloca-se uma LC de CB média e avalia-se à lâmpada de fenda. A LC deve ter, preferencialmente, diâmetro grande para cobrir toda a córnea e mobilidade ligeiramente abaixo do normal para causar o menor traumatismo possível. Após a colocação da LC deve ocorrer a melhora dos sintomas.

O controle e a manutenção das LCT - O ideal é que todo paciente adaptado com LCT seja examinado no dia seguinte para rever o padrão de adaptação e pesquisar sinais de inflamação ocular. Se a LC estiver muito apertada terá que ser substituída por outra mais plana. Se apresentar mobilidade excessiva, deve ser trocada por CB mais apertada, pois pode causar desconforto e trauma na córnea. Se houver suspeita de infecção ou reação inflamatória muito intensa, o uso deve ser interrompido.

O controle poderá ser diário, semanal ou quinzenal, dependendo da gravidade do problema ocular que determinou a adaptação da LC. Durante o exame de controle, se for necessário realizar a tonometria, de modo geral, retira-se a LCT com cuidado e depois da tonometria, antes de recolocá-la, lava-se o olho com soro para remover a fluoresceína. Trabalho publicado por Allen, comparando a medida da pressão intra-ocular, com ou sem a LC, em usuários de LCT de silicone-hidrogel, realizada com o tonômetro de aplanação de Goldmann, mostrou que não houve diferença entre as medidas $^{(4)}$. A tonometria com aparelhos de não-contato pode ser feita sem a remoção da LCT.

A remoção para a manutenção ou a substituição da LC depende do estágio da doença e é determinada pelo médico. As LCT de hidrogel podem ser usadas no máximo por uma semana e as de silicone-hidrogel por até 30 dias. A substituição freqüente reduz as complicações derivadas da formação de depósitos, mas aumenta o risco de trauma corneal.

A instilação de colírios sobre a LCT pode ser necessária. Indicam-se medicamentos sem preservativos para evitar toxicidade e reações de sensibilidade, principalmente quando se trata do uso freqüente de lágrimas artificiais. Soluções hipertônicas podem causar desidratação da LCT e síndrome de LC apertada, por isso devem ser evitadas ${ }^{(5)}$.

As complicações mais freqüentes são: neovascularização corneal, infiltrados estéreis, edema, úlcera de córnea infecciosa, hipópio, iridociclite e conjuntivite papilar gigante (CPG). Pacientes com história prévia de CPG devem usar LC com material crofilcon (p-MMA/GMA) metacrilato de glicerol, que apresenta baixa incidência desta complicação( ${ }^{(5)}$, ou outro material não iônico com baixa hidratação.

A ocorrência de úlcera de córnea infecciosa é mais freqüente entre usuários de LCT do que entre aqueles que usam LC apenas para corrigir vícios de refração. As LCT são adaptadas em olhos doentes, ou submetidos à cirurgia e, por isso, predispostos à complicações.

\section{Considerações sobre LCT de silicone-hidrogel}

Por permitir maior transmissibilidade ao $\mathrm{O}_{2}$ do que o material hidrofílico, o de silicone-hidrogel apresenta maior facili- dade de recuperação do epitélio corneal; menor edema epitelial ou estromal. Entretanto, a interação mecânica com o tecido ocular e os efeitos sobre a fisiologia e estrutura do filme lacrimal são similares aos encontrados nos outros tipos de $\mathrm{LC}^{(6)}$.

LC de silicone-hidrogel pode ser usada com fins terapêuticos por até 30 dias sem remoção, embora seja preferível descartá-la em 15 dias $^{(7)}$. Isso beneficia pacientes que devem usar LCT por mais de 7 dias, porque evita-se inserção e remoção freqüentes, que podem estar associadas com o sintoma da dor causada pelo trauma epitelial. A LC de silicone-hidrogel por ser mais resistente à formação de depósitos do que a hidrofílica apresenta menor risco de complicação ocular. O aumento de permeabilidade ao $\mathrm{O}_{2}$ proporcionado pelo material silicone-hidrogel pode reduzir mas não eliminar o risco de ceratite infecciosa ${ }^{(8-9)}$.

Estudos com LCT de silicone-hidrogel mostraram recuperação de alterações corneais em $74 \%$ a $91 \%$ dos casos e $78 \%$ a $94 \%$ dos pacientes relataram alívio da dor ${ }^{(10-11)}$.

Um estudo comparativo entre LC de silicone-hidrogel e hidrofílicas, usadas como terapêuticas após LASEK, mostrou que depois de 5 dias não havia diferença na hiperemia conjuntival e límbica, na quantidade de debris aderidos às LC ou no conforto subjetivo dos pacientes, em qualquer visita pósoperatória. Entretanto, o processo de restabelecimento do epitélio corneal apresentou melhora estatisticamente significante $(\mathrm{P}=0,01)$ nos olhos com LC silicone-hidrogel ${ }^{(12)}$.

A opção não deve ser LCT de silicone-hidrogel quando o objetivo é proteger a córnea e estimular a vascularização estromal, em olhos sem prognóstico visual. Nesses casos, utiliza-se LC hidrofílica espessa, de baixo conteúdo de água ${ }^{(5)}$.

\section{CONCLUSÃO}

As LC silicone-hidrogel causam menor incidência de complicações associadas à hipóxia. A vantagem de permitir maior tempo de uso sem remoção diminui o trauma epitelial, a dor, e o potencial de infecção, além de reduzir o custo do tratamento.

O paciente deve ser orientado quanto aos objetivos e benefícios das LC terapêuticas e, também, informado sobre os riscos.

\section{ABSTRACT}

Therapeutic contact lenses are useful in a variety of ocular surface diseases. Their main indications are: to relieve the pain; protect ocular surface; promote corneal healing and epithelial regeneration; seal a leaking corneal wound and deliver ophthalmic drugs on the ocular surface. There are several kinds of lens designs and materials, and their choice is dependent on the specific disease to be treated, the duration of treatment and the physiologic needs of the diseased cornea. Bullous keratopathy, recurrent epithelial erosion syndrome, dry eye and 
postoperative epithelial defects are amongst their indications. Therapeutic contact lenses should not be indicated in the presence of active infectious keratitis or when the patient is not compliant. Corneal neovascularization, giant papillary conjunctivitis and infectious keratitis are serious complications, which can be prevented by correctly fitting and maintaining the therapeutic contact lenses. Silicon-hydrogel therapeutic contact lenses, due to their higher oxygen permeability, allow extended wear schedules, decreasing the need for frequent lens replacement.

Keywords: Contact lenses, hydrophilic; Hydrogels; Silicones; Epithelium, corneal/physiology; Wound healing/physiology; Keratitis; Photorefractive keratectomy; Visual acuity

\section{REFERÊNCIAS}

1. Russo PA, Bouchard CS, Galasso JM. Extended-wear silicone hydrogel soft contact lenses in the management of moderate to severe dry eye signs and symptoms secondary to graft-versus-host disease. Eye Contact Lens. 2007; 33(3):144-7.
2. Schafer J, Mitchell GL, Chalmers RL, Long B, Dillehay S, Barr J, et al. The stability of dryness symptoms after refitting with silicone hydrogel contact lenses over 3 years. Eye Contact Lens. 2007;33(5):247-52.

3. Schrader S, Wedel T, Moll R, Geerling G. Combination of serum eye drops with hydrogel bandage contact lenses in the treatment of persistent epithelial defects. Graefes Arch Clin Exp Ophthalmol. 2006;244(10):1345-9.

4. Allen RJ, Dev Borman A, Saleh GM. Applanation tonometry in silicone hydrogel contact lens wearers. Cont Lens Anterior Eye. 2007;30(5):267-9.

5. Foulks GN, Harvey T, Raj CV. Therapeutic contact lenses: the role of highDk lenses. Ophthalmol Clin North Am. 2003;16(3):455-61.

6. Stapleton F, Stretton S, Papas E, Skotnitsky C, Sweeney DF. Silicone hydrogel contact lenses and the ocular surface. Ocul Surf. 2006;4(1):24-43.

7. Gürdal C, Takmaz T, Sargon MF, Anayol A, Ylmaz E, Can I. Electron microscopic evaluation of the effect of therapeutic silicone hydrogel lenses on the limbal area. Eye Contact Lens. 2006;32(3):133-7.

8. Kanpolat A, Uçakhan OO. Therapeutic use of Focus Night \& Day contact lenses. Cornea. 2003;22(8):726-34.

9. Schornack MM, Faia LJ, Griepentrog GJ. Pseudomonas keratitis associated with daily wear of silicone hydrogel contact lenses. Eye Contact Lens. 2008; $34(2): 124-8$.

10. Montero J, Sparholt J, Mély R, Long B. Retrospective case series of therapeutic applications of lotrafilcon a silicone hydrogel soft contact lenses. Eye Contact Lens. 2003;29(2):72-5.

11. Ambroziak AM, Szaflik JP, Szaflik J. Therapeutic use of a silicone hydrogel contact lens in selected clinical cases. Eye Contact Lens. 2004;30(1):63-7.

12. Gil-Cazorla R, Teus MA, Arranz-Márquez E. Comparison of silicone and non-silicone hydrogel soft contact lenses used as a bandage after LASEK. J Refract Surg. 2008;24(2):199-203. 